\title{
The component retrieval problem in printed circuit board assembly
}

Citation for published version (APA):

Spieksma, F. C. R., Crama, Y., van de Klundert, J., \& Flippo, O. E. (1995). The component retrieval problem in printed circuit board assembly. METEOR, Maastricht University School of Business and Economics. METEOR Research Memorandum No. 027 https://doi.org/10.26481/umamet.1995027

Document status and date:

Published: 01/01/1995

DOI:

10.26481/umamet.1995027

Document Version:

Publisher's PDF, also known as Version of record

\section{Please check the document version of this publication:}

- A submitted manuscript is the version of the article upon submission and before peer-review. There can be important differences between the submitted version and the official published version of record.

People interested in the research are advised to contact the author for the final version of the publication, or visit the DOI to the publisher's website.

- The final author version and the galley proof are versions of the publication after peer review.

- The final published version features the final layout of the paper including the volume, issue and page numbers.

Link to publication

\footnotetext{
General rights Owners
rights.

- You may freely distribute the URL identifying the publication in the public portal. please follow below link for the End User Agreement:

www.umlib.nl/taverne-license

Take down policy

If you believe that this document breaches copyright please contact us at:

repository@maastrichtuniversity.nl

providing details and we will investigate your claim.
}

Copyright and moral rights for the publications made accessible in the public portal are retained by the authors and/or other copyright owners and it is a condition of accessing publications that users recognise and abide by the legal requirements associated with these

- Users may download and print one copy of any publication from the public portal for the purpose of private study or research.

- You may not further distribute the material or use it for any profit-making activity or commercial gain

If the publication is distributed under the terms of Article $25 \mathrm{fa}$ of the Dutch Copyright Act, indicated by the "Taverne" license above, 


\title{
The Component Retrieval Problem in Printed Circuit Board Assembly
}

\author{
Yves Crama ${ }^{1}$ \\ Olaf E. Flippo ${ }^{2}$ \\ Joris van de Klundert ${ }^{2}$ \\ Frits C.R. Spieksma ${ }^{3}$
}

October 16, 1995

\footnotetext{
${ }^{1}$ Ecole d'Administration des Affaires, Université de Liège, 4000 Liège, Belgium

${ }^{2}$ Department of Quantitative Economics, Faculty of Economics, University of Limburg, 6200 MD Maastricht, The Netherlands

${ }^{3}$ Department of Mathematics, University of Limburg, 6200 MD Maastricht, The Netherlands
} 


\begin{abstract}
The minimization of the makespan of a printed circuit board assembly process is a complex problem. Decisions involved in this problem concern the specification of the order in which components are to be placed on the board, and the assignment of component types to the feeder slots of the placement machine. If some component types are assigned to multiple feeder slots, then the additional problem emerges of selecting, for each placement on the board, the feeder slot from which the related component type is to be retrieved. In this paper, we consider this Component Retrieval Problem for placement machines that operate in a similar way as the Fuji CP II. We explain why a simple forward dynamic programming scheme cannot provide an efficient solution to this problem, thereby invalidating the correctness of an earlier published approach. We then present a polynomial algorithm that solves the problem to optimality.

The analysis of the Component Retrieval Problem is greatly facilitated by its reformulation as a longest path problem in a PERT/CPM network with design aspects; finding the minimal makespan of the assembly process thus amounts to identifying a design for which the longest path in the induced network is shortest. As an alternative interpretation, the Component Retrieval Problem can be viewed as a shortest path problem with side-constraints. The complexity of these network problems is analysed, and it is proven that the polynomial solvability of the Component Retrieval Problem is caused by the specific structure it inflicts on the arc lengths in the network. In the absence of this structure, the network problems are shown to be $N P$-hard in general.
\end{abstract}




\section{Introduction}

The problem of determining optimal production plans for the automated assembly of printed circuit boards (PCBs) has been investigated by numerous researchers; see e.g. Ahmadi (1993) and Crama, Oerlemans and Spieksma (1994). A precise definition of this problem is highly dependent on the specific features of the assembly machines and, more generally, of the technological environment. As a rule, however, the problem is a very complex one and, for this reason, many authors have proposed to solve it by decomposing it into subproblems; see e.g. Ahmadi (1993), Ball and Magazine (1988), Bard, Clayton and Feo (1994), Crama, Flippo, van de Klundert and Spieksma (1995), Crama, Oerlemans and Spieksma (1994) and van Laarhoven and Zijm (1993). Here again, the subproblems emerging from the decomposition vary according to the context, as do their computational complexity. In this paper, we concentrate on one such subproblem, namely the Component Retrieval Problem (CRP) that arises when the placement machine operates like a machine of the Fuji CP family. Briefly stated, CRP is defined to be the following problem: for a given placement sequence of components on the board, and for a given assignment of component types to (possibly multiple) feeder slots of the placement machine, decide from which feeder slot each component should be retrieved.

In the next section, we describe the assembly process associated with a Fuji CP II placement machine, and the role of the Component Retrieval Problem in this process. We refer to Bard, Clayton and Feo (1994) and Crama et al. (1995) for a more complete description of this process as well as for related references. In Section 3, we present a formulation of CRP in terms of a PERT/CPM network problem with design aspects; finding the minimal makespan of the assembly process thus amounts to identifying a design for which the longest path in the induced network is shortest. Alternatively, the Component Retrieval Problem may also be viewed as a shortest path problem with (path induced) side-constraints. In Section 4 an example is discussed which reveals that straightforward forward dynamic programming does not necessarily yield optimal solutions. In fact, the example suggests that no dynamic programming approach with a linearly sized state-space in the number of components involved, is capable of retaining sufficient information to identify optimal solutions in all cases. These negative observations, which also invalidate the forward dynamic programming approach by Bard, Clayton and Feo (1994), may serve as a justification for the relatively complex solution algorithm that is proposed in Section 5. This algorithm is based on the network formulation of Section 3, and can be viewed as a "two-phase" dynamic programming approach with pairs of grip activities as the state-space. If the number of components involved is denoted by $n$, then the size of the state-space is thus quadratic in $n$, implying an overall time complexity of $O\left(n^{3}\right)$ for the entire algorithm.

Since the network optimization problems of Section 3 are of interest beyond the special case of CRP, their time complexity is studied in Section 6. It is proven that the polynomial solvability of the Component Retrieval Problem is caused by the specific structure it inflicts on the arc lengths in the PERT/CPM network; in the absence of this structure, the network problem is shown to be $N P$-hard in general. The paper is concluded with a brief summary. 


\section{The Fuji CP II Placement Machine}

Assembling a printed circuit board consists of placing a number of electronic components, each of prespecified type, at prespecified locations on a bare board. The placement machine that is considered in this paper is a Fuji CP II, yet our analysis may apply to other machines having similar characteristics as well (such as other members of the Fuji family, or the Panasonic Mk1 considered by Horak and Francis (1995)). The Fuji CP II is equipped with a magazine rack that contains a number of slots to which feeder tapes can be assigned. Each tape bears components of a unique component type, and feeder tapes with the same component type may be assigned to multiple slots. ${ }^{1}$ Components are gripped from a slot of the magazine rack and mounted on the PCB by a placement head. Coordination between grip and place activities is done by a carousel, which performs many other functions as well. The carousel contains 12 heads, and it can simultaneously hold up to six components; see Figure 1.

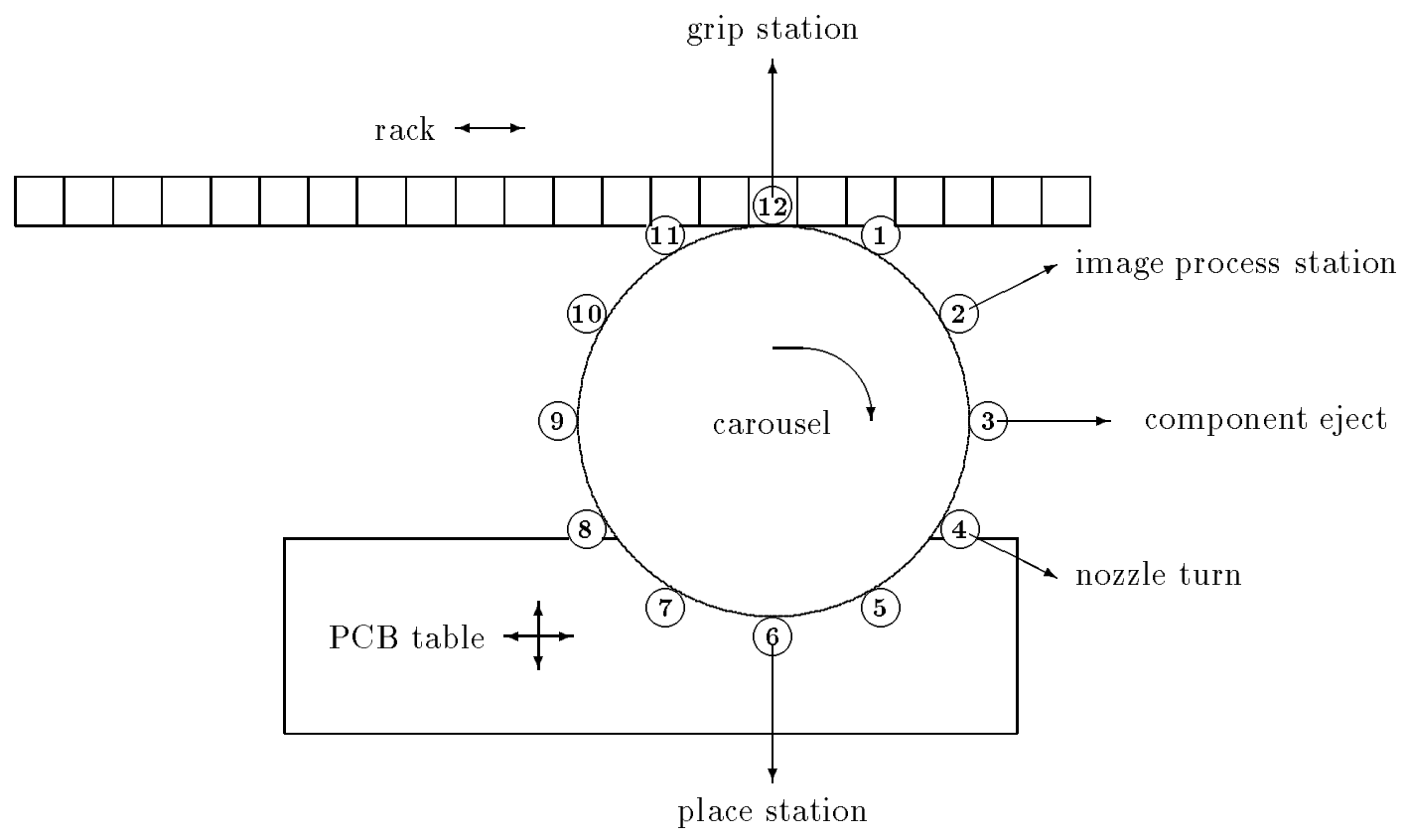

Figure 1: The Fuji CP II.

Suppose the machine is just about to place the $i$-th component on the board. To this end, the board location where the component is to be placed, is positioned at the so-called "placement spot", and the carousel head containing the component (the current place station) is right above this spot. The carousel head then proceeds with the actual placement of the component on the board. After the placement has been completed, the worktable holding the PCB starts moving, until the board location where the next component is to be placed comes to rest at the placement spot. Diametrically opposed to the aforementioned carousel head is another head (the current grip station), which is positioned right above the so-called "gripping spot". The grip station is ready to grip the $(i+6)$-th component from the magazine rack as soon as the appropriate slot has been positioned at the gripping spot.

\footnotetext{
${ }^{1}$ In fact, it will become clear shortly that a non-trivial instance of the Component Retrieval Problem only emerges if at least one component type is assigned to at least two different feeder slots.
} 
Once this is done, the head proceeds with actually gripping the $(i+6)$-th component from this feeder slot. After the gripping activity has been completed, the magazine rack starts to shift in order to position the slot from which the next component is to be retrieved, at the gripping spot. Only after the $i$-th component has been placed and the $(i+6)$-th component has been gripped, the carousel is ready to rotate $30^{\circ}$ clockwise, to prepare for the placement and gripping of components $(i+1)$ and $(i+7)$ respectively.

Thus, between two consecutive place activities, the PCB table has to move until the board location where the second component is to be placed lies at the placement spot, and the carousel has to rotate $30^{\circ}$ so as to position the next head right above the placement spot. Similarly, between two consecutive grip activities, the rack has to shift until the appropriate slot is at the gripping spot, and the carousel has to rotate so as to position the next head right above this spot. It is hereby important to observe that placement operation $i$ and gripping operation $(i+6)$ do not have to be performed simultaneously, but are necessarily carried out between the same two carousel rotations. Also, table, rack and carousel movements may take place concurrently.

Clearly, the duration of rack movements depends on the distance between slots from which consecutive gripping operations are done. Therefore, even when the component placement sequence on the board and the magazine rack assignment of component tapes are given, minimizing the assembly makespan still involves decisions concerning the feeder slots from which each component should be retrieved. The corresponding optimization problem is known as the Component Retrieval Problem (CRP). Of course, as mentioned before, a non-trivial decision problem only arises if at least one component type is assigned to at least two different feeder slots. This type of feeder duplication is also discussed in e.g. Ahmadi, Grotzinger and Johnson (1988), Bard, Clayton and Feo (1994) and Tang and Denardo (1988).

\section{The Component Retrieval Problem as a PERT/CPM Network Model with Design Aspects}

In order to facilitate our discussion, we present a PERT/CPM-like model of CRP. To achieve this, we first need to introduce several assumptions and develop some notation. First, let $1, \ldots, n$ denote the components that are to be mounted on the PCB, with the numbering reflecting their placement sequence on the board. With respect to the starting conditions of the assembly process, we assume that the feeder slot from which the first component will be retrieved, is initially positioned below the grip station (currently occupied by carousel head 12), and that the PCB location where the first place activity will occur, is initially positioned below the place station (currently occupied by carousel head 6). Furthermore, components $1-6$ have been added as fictitious components, initially held by carousel heads $6-1$ respectively; they are to be mounted at the same board location as component 7 , which operation can be performed in zero time. If we similarly assume that 6 fictitious and instantaneous grip activities are carried out at the end of the mounting process, then a situation has been constructed where exactly $n$ grip activities and $n$ place activities are required to assemble the board, with the $i$-th grip and $i$-th place activity occurring between the $(i-1)$-st and $i$-th carousel rotation.

As a first step towards modeling CRP, let us briefly explain how, for a given solution $S$ of CRP, the 


\begin{tabular}{|lll|}
\hline \hline$g_{i}=$ start of the $i$-th grip activity & $(i=1, \ldots, n)$ \\
$p_{i}$ & $=$ start of the $i$-th place activity & $(i=1, \ldots, n)$ \\
$\Delta g_{i}$ & $=$ duration of the $i$-th grip activity & $(i=1, \ldots, n)$ \\
$\Delta p_{i}$ & $=$ duration of the $i$-th place activity & $(i=1, \ldots, n)$ \\
$\Delta m_{i}=$ duration of the $i$-th rack movement & $(i=1, \ldots, n-1)$ \\
$\Delta t_{i}=$ duration of the $i$-th table movement & $(i=1, \ldots, n-1)$ \\
$\Delta c_{i}=$ duration of the $i$-th carousel rotation & $(i=1, \ldots, n-1)$ \\
\hline \hline
\end{tabular}

Table 1: Events and activities of the PERT/CPM graph $D(S)$

assembly makespan can be computed by classical PERT/CPM techniques. To this end, the events (i.e. moments in time) and activities (i.e. time durations) of Table 1 are introduced. Events, activities and precedence relations between activities can be represented by a PERT/CPM graph $D(S)$ (recall that $S$ is the given solution to CRP), where nodes and arcs correspond to events and activities respectively, and arc lengths denote activity durations; see Figure 2. We will refer to the nodes as grip or place nodes, depending on the nature of the associated event. The resulting graph consists of $n$ layers, where each layer $i$ contains exactly one grip node $g_{i}$ and one place node $p_{i}(i=1, \ldots, n)$. To model the start and the end of the assembly process, it is convenient to add a source, indifferently denoted by $p_{0}$ and $g_{0}$, and a sink, indifferently denoted by $p_{n+1}$ and $g_{n+1}$. As is well-known, the makespan of the assembly process is equal to the length of a longest path in $D(S)$ from $p_{0}$ to $p_{n+1}$. Computing a longest path in such an acyclic and layered network can be done by forward dynamic programming in $O(n)$ time (see e.g. Ahuja, Magnanti and Orlin (1993)).

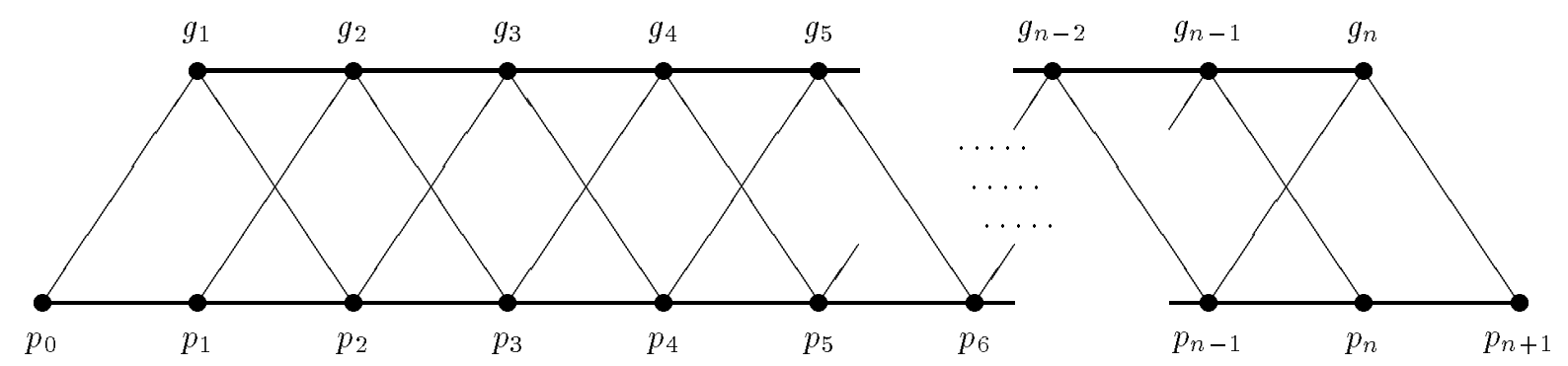

Figure 2: The PERT/CPM graph $D(S)$

In order to specify the arc lengths of $D(S)$, recall from Section 2 that between the start of two consecutive grip activities $g_{i}$ and $g_{i+1}(i=1, \ldots, n-1)$, the following operations have to be performed: the $i$-th grip activity, the $i$-th carousel rotation and the $i$-th rack movement. Since the former precedes the latter two, and the latter two may be carried out concurrently, it follows that the length of arc $\left(g_{i}, g_{i+1}\right)$ equals $\Delta g_{i}+\max \left\{\Delta c_{i}, \Delta m_{i}\right\}$. On the other hand, the $(i+1)$-st grip activity can only start when both the $i$-th place activity and the $i$-th carousel rotation are completed. Since these activities are carried out consecutively, it follows that the length of arc $\left(p_{i}, g_{i+1}\right)$ equals $\Delta p_{i}+\Delta c_{i}$. Other arc lengths in $D(S)$ are defined in a similar fashion; see Table 2. 


\begin{tabular}{|l|l|l|}
\hline \hline arc & for & length \\
\hline$\left(g_{i}, g_{i+1}\right)$ & $i=1, \ldots, n-1$ & $\Delta g_{i}+\max \left\{\Delta c_{i}, \Delta m_{i}\right\}$ \\
$\left(p_{i}, p_{i+1}\right)$ & $i=0, \ldots, n$ & $\Delta p_{i}+\max \left\{\Delta c_{i}, \Delta t_{i}\right\}$ \\
$\left(g_{i}, p_{i+1}\right)$ & $i=1, \ldots, n$ & $\Delta g_{i}+\Delta c_{i}$ \\
$\left(p_{i}, g_{i+1}\right)$ & $i=0, \ldots, n-1$ & $\Delta p_{i}+\Delta c_{i}$ \\
\hline \hline
\end{tabular}

Table 2: Arc lengths of $D(S)$ with $\Delta c_{i}=\Delta t_{i}=0$ for $i=0, n$ and $\Delta p_{0}=0$

In view of the above discussion, the Component Retrieval Problem can now be modelled as follows. Consider the graph of Figure 2. For each $i=1, \ldots, n$, we introduce a set of grip nodes $G_{i}$ instead of only one grip node $g_{i}$, where each node of $G_{i}$ refers to one of the slots containing the component type required for the $i$-th grip activity. Figure 3 shows an example where the first two components (which may or may not be of the same type) can both be retrieved from two alternative feeder slots, and all other components can only be retrieved from one such slot. Then specifying a component retrieval plan, i.e. a feasible solution $S$ of CRP, amounts to selecting exactly one grip node from each set $G_{i}$, in such a way that the longest path in the subgraph induced by the selected nodes be as short as possible. We thus arrive at the following formalization of CRP graphs and problems.

Definition 1 (CRP graph) A CRP graph $D=(V, A)$ is a layered directed graph on the node set $V=\cup_{i=0}^{n+1} L_{i}$, with the layers $L_{i}$ being mutually disjoint sets. Moreover, $L_{i}=\left\{p_{i}\right\} \cup G_{i}$, where $G_{i}$ is a non-empty set not containing $p_{i}(i=1, \ldots, n)$ and $G_{0}=G_{n+1}=\emptyset$. The set $\left\{p_{0}, \ldots, p_{n+1}\right\}$ is referred to as the set of place nodes; all other nodes are called grip nodes. The arc set $A$ is given by $A=\left\{(u, v) \mid u \in L_{i}, v \in L_{i+1}\right.$ for some $\left.i=0, \ldots, n\right\}$, with the length of arc $(u, v)$ being denoted by $d(u, v)$. The length function $d(\cdot)$ satisfies

$$
d\left(g_{i}, p_{i+1}\right)+d\left(p_{i}, g_{i+1}\right) \leq d\left(g_{i}, g_{i+1}\right)+d\left(p_{i}, p_{i+1}\right) .
$$

for all $g_{i} \in G_{i}, g_{i+1} \in G_{i+1}$ and $i=1, \ldots, n-1$.

Note that the arc lengths displayed in Table 2 satisfy inequality (1). In the sequel (with the exception of Theorem 1), we will not make any explicit use of the specific lengths displayed in Table 2, but we will rely on their property (1) instead. We will see in Sections 5 and 6 that this property guarantees the efficient solvability of CRP. It may also be interesting to remark that the inequalities (1) are somewhat reminiscent of a matrix property studied in the literature under the name 'Monge property' (see e.g. Burkard, Klinz and Rudolf (1995)).

Definition 2 (Selection) A selection $S$ in a CRP graph $D$ is a set of grip nodes containing exactly one grip node from each layer, i.e. $\left|S \cap G_{i}\right|=1$ for $i=1, \ldots, n$.

Definition 3 (Selection induced subgraph) For any selection $S$ in a $C R P$ graph $D$, the selection induced subgraph $D(S)=(V(S), A(S))$ is the subgraph of $D$ that is induced by $S \cup\left\{p_{0}, \ldots, p_{n+1}\right\}$. The length of a longest path in $D(S)$ is denoted by $L(D(S))$. 
Since the length of a longest path in the subgraph induced by selection $S$ is equal to the makespan of the PCB assembly process using the retrieval plan defined by $S$, we arrive at the following network version of the Component Retrieval Problem.

Definition 4 (CRP Problem) Given a CRP graph D, the Component Retrieval Problem is to determine a selection $S$ which minimizes $L(D(S))$, i.e. the length of a longest path in $D(S)$.

As mentioned before, the analysis and results in this paper will all apply to this network version of the Component Retrieval Problem, and not only to the special instances arising from the original application where arc lengths are defined as in Table 2.

The above definitions reveal that CRP is basically a PERT/CPM network problem with design aspects. Obviously, designs are restricted to selections in this case, i.e. they must contain exactly one grip node per layer. As an alternative interpretation, the minimization of the makespan seems to indicate that all grip activities should be completed as early as possible. This, in its turn, seems to suggest that a minimal makespan can be obtained by computing a shortest path from $p_{0}$ to $p_{n+1}$ in the subgraph that is induced by these two place nodes and all grip nodes (the so-called "grip graph"). Unfortunately, the precedence relations that are induced by the place activities would be completely ignored in such an approach; a shortest path through the grip graph would only specify an optimal selection if, between each pair of grip nodes, the makespan (longest path length) that results from the interfering place activities (nodes) was taken into consideration as a lower bounding side-constraint. Therefore, CRP can also be viewed as a shortest path problem with side-constraints. Obviously, the side-constraints are of a very specific nature here, viz. they result from longest path lengths induced by a single (place) path that is added to the (grip) graph under consideration. The aforementioned two interpretations of CRP are interesting in their own right. In Section 6 it will be shown that although CRP can be solved in polynomial time, more general versions of the problem probably cannot, since the absence of the arc length structure (1) makes them NP-hard in general.

\section{CRP and forward dynamic programming}

As briefly mentioned in the introduction, the Component Retrieval Problem has been previously investigated by Bard, Clayton and Feo (1994), who proposed a forward dynamic programming scheme for its solution. Before we present our algorithm for CRP, we deem it necessary to explain why the approach proposed by Bard, Clayton and Feo cannot possibly lead to a correct algorithm for CRP.

Consider the CRP graph of Figure 3. Let the arc lengths of $\left(g_{1}^{1}, g_{2}^{2}\right)$ and $\left(g_{1}^{2}, g_{2}^{1}\right)$ be "large" (say, larger than 10), and let all arcs $\left(p_{i-1}, g_{i}\right)$ and $\left(g_{i}, p_{i+1}\right)(i=1, \ldots, n)$ have length zero. The other arc lengths are as indicated in Figure 3 . Note that $\left(g_{n-1}, g_{n}\right)$ has length $4+x$. In the upcoming discussion, we will consider two possible values for $x$, viz. $x=0$ and $x=5$ respectively.

Since $d\left(g_{1}^{1}, g_{2}^{2}\right)$ and $d\left(g_{1}^{2}, g_{2}^{1}\right)$ are "large", the only candidate optimal selections are $S^{1}=$ $\left\{g_{1}^{1}, g_{2}^{1}, g_{3}, \ldots, g_{n}\right\}$ and $S^{2}=\left\{g_{1}^{2}, g_{2}^{2}, g_{3}, \ldots, g_{n}\right\}$. Table 3 displays the longest path (length) in the corresponding selection induced subgraphs, for $x=0$ and $x=5$ respectively. Observe that $S^{1}$ is optimal when $x=0$, whereas $S^{2}$ is optimal when $x=5$. 


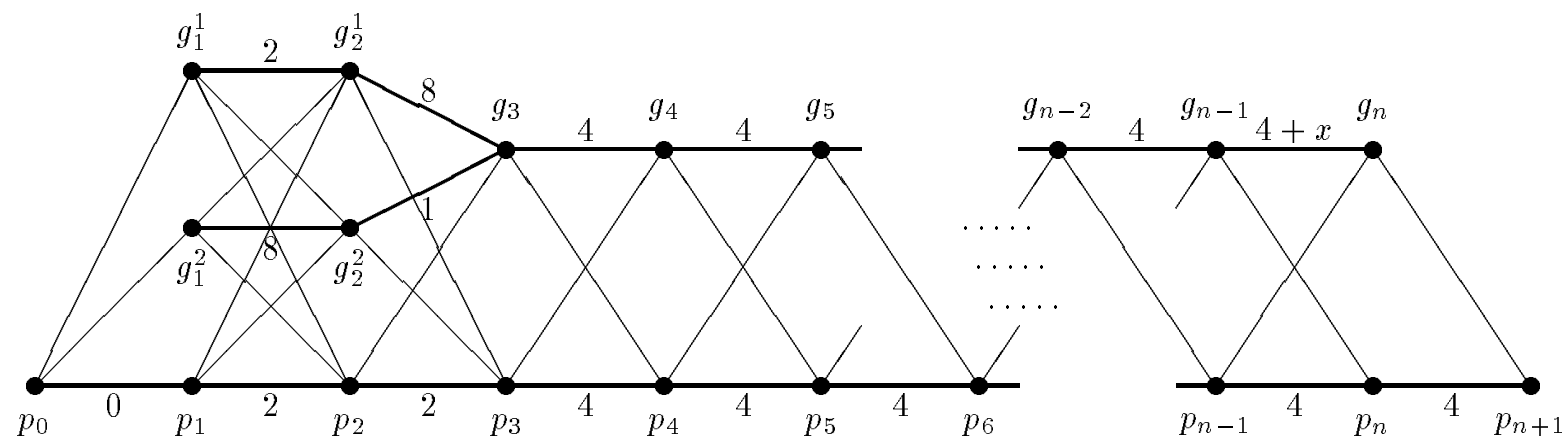

Figure 3: A counterexample for straightforward dynamic programming

\begin{tabular}{||c||l|l||}
\hline \hline Longest Paths & \multicolumn{1}{|c||}{$S^{1}$} & \multicolumn{1}{c||}{$S^{2}$} \\
\hline \hline$x=0$ & $\left\{p_{0}, g_{1}^{1}, g_{2}^{1}, g_{3}, \ldots, g_{n}, p_{n+1}\right\}$ & $\left\{p_{0}, g_{1}^{2}, g_{2}^{2}, p_{3}, \ldots, p_{n}, p_{n+1}\right\}$ \\
& length: $10+4(n-3)$ & length: $12+4(n-3)$ \\
\hline$x=5$ & $\left\{p_{0}, g_{1}^{1}, g_{2}^{1}, g_{3}, \ldots, g_{n}, p_{n+1}\right\}$ & $\left\{p_{0}, g_{1}^{2}, g_{2}^{2}, g_{3}, \ldots, g_{n}, p_{n+1}\right\}$ \\
& length: $15+4(n-3)$ & length: $14+4(n-3)$ \\
\hline \hline
\end{tabular}

Table 3: Longest path (length) under different scenarios

A simple forward dynamic programming scheme for CRP, with the set of grip nodes as state space, would be based on the following definition:

$$
\phi\left(g_{i}\right)=\text { minimum length of a longest path from } p_{0} \text { to } g_{i} \text {. }
$$

Note that $\phi\left(g_{i}\right)=9+4(i-3)$ for $3 \leq i \leq n-1$, with the optimal predecessor of $g_{i}$ being $g_{2}^{2}$. However, when $x=0$, then the unique optimal selection, i.e. $S^{1}$, does not contain $g_{2}^{2}$. Similarly, if the place nodes form the state space, that is

$$
\psi\left(p_{i}\right)=\text { minimum length of a longest path from } p_{0} \text { to } p_{i},
$$

then $\psi\left(p_{i}\right)=6+4(i-3)$ for $4 \leq i \leq n$, with the optimal predecessor of $p_{i}$ being $g_{2}^{1}$. Yet again, when $x=5$, then $g_{2}^{1}$ is not part of the optimal selection $S^{2}$. These observations clearly show that the Principle of Optimality does not hold in either case: in order to identify an optimal selection for the entire problem, it may be necessary to keep track of partial selections that are non-optimal up to certain layers.

In addition, the information that is required to track the first part of an optimal selection for the entire problem may be contained in arbitrarily remote parts of the graph, even as remote as the very last grip arc. The conclusion is that simple forward dynamic programming does not necessarily identify optimal selections, not even if the recursion is equipped with a "look-k-layers-ahead-or-back" capability for constant $k$. To guarantee optimality, a more elaborate analysis and approach therefore seems to be required. 
Let us stress that these negative conclusions directly affect the validity of the forward dynamic programming algorithm proposed by Bard, Clayton and Feo (1994). Indeed, the recursive formulation considered by these authors reads:

$$
f_{i+1}(k)=\min _{j \in Y_{i}}\left\{t_{j k}(i, i+1)+f_{i}(j)\right\} \quad k \in Y_{i+1}, i=0,1, \ldots, n-1
$$

where

$$
\begin{aligned}
f_{i}(k)= & \begin{array}{l}
\text { minimum time required to grip the first } i \text { components given that } \\
\text { the } i \text {-th component is retrieved from magazine slot } k ;
\end{array}= \\
Y_{i} & \begin{array}{l}
\text { set of magazine slots containing the component type required for } \\
\text { the } i \text {-th gripping activity; }
\end{array} \\
t_{j k}(i, i+1)= & \text { elapsed time between completion of the } i \text {-th gripping activity from } \\
& \text { slot } j \text { and the }(i+1) \text {-st gripping activity from slot } k .
\end{aligned}
$$

Note that the interpretation of $f_{i}(k)$ coincides with that of $\phi\left(g_{k}\right)$ (see above), if $g_{k}$ is the node associated with feeder slot $k$ in layer $L_{i}$ of the CRP graph. What the example of this section shows (and what seems to have been overlooked in Bard, Clayton and Feo (1994)) is that, in an optimal retrieval plan where the $i$-th component is retrieved from slot $k$, the time required to grip the first $i$ components may strictly exceed $f_{i}(k)$ for some $i$ and $k$. An alternative way of understanding this conclusion is to realize that the time interval $t_{j k}(i, i+1)$ is not univoquely determined by $j, k$ and $i$ (contrary to what its notation suggests), but actually depends on the sequence of grip activities prior to the $i$-th one. This explains why the recursion (2) does not lead to an easy algorithm for CRP.

For the sake of completeness, let us add some more comments on a variant of CRP which we encountered in a practical setting, and which can be solved efficiently by forward dynamic programming. In Crama et al. (1995), we describe an industrial case study in which the operating mode of the placement machine has been restricted as follows: for each $i=1, \ldots, n$, the start of the $i$-th placement operation is required to coincide with the start of the $(i+6)$-th gripping operation (in contrast with the description given in Section 2). It is easy to see that recursion (2) is valid under this restriction. Indeed, the value of $t_{j k}(i, i+1)$ can now be simply expressed as

$$
t_{j k}(i, i+1)=\max \left\{\Delta g_{i}+\Delta c_{i}, \Delta g_{i}+\Delta m_{j k}, \Delta p_{i}+\Delta c_{i}, \Delta p_{i}+\Delta t_{i}\right\}
$$

(where $\Delta m_{j k}$ is the duration of the rack movement from slot $j$ to slot $k$, and the other notations have been previously defined). In particular, since the expression (3) only depends on $j, k$ and $i$, the assembly makespan can be computed in $O(n)$ time by solving recursion (2) (see Crama et al. (1995)). In view of the relative simplicity of this procedure (as compared to the algorithm described in the next section), one may rightfully wonder to what extent the makespan of the selection that it delivers differs from the optimal makespan computed for the unrestricted machine.

More formally, for an arbitrary PCB, let $S$ denote an optimal solution (viz., selection, or retrieval plan) of CRP, and let $U=L(D(S))$ denote the optimal assembly makespan of this PCB on a 
placement machine operating in unrestricted mode. Similarly, let $S^{\text {res }}$ denote an optimal retrieval plan for the same PCB when the machine operates in restricted mode (viz., $S^{\text {res }}$ is the solution of (2) when $t_{j k}(i, i+1)$ is defined by $(3)$ ), denote by $R$ the makespan of $S^{\text {res }}$ on the restricted machine and denote by $H=L\left(D\left(S^{\text {res }}\right)\right)$ the makespan of $S^{\text {res }}$ on the unrestricted machine. Thus, $R$ is the optimal assembly makespan for the restricted machine, whereas $H$ (which stands for 'heuristic') is the makespan obtained on the unrestricted machine when we use the retrieval plan $S^{\text {res }}$ rather than the optimal plan $S$. We are interested in the maximal value that can be achieved by each of the ratios $R / U$ and $H / U$ (notice that $R / U$ measures the productivity loss that results from using the machine in restricted mode, whereas $H / U$ measures the loss that results from using the suboptimal plan $S^{r e s}$ rather than $S$ ). Under the (realistic) assumption that all data $\Delta g_{i}, \Delta p_{i}, \Delta c_{i}, \Delta t_{i}$ and $\Delta m_{j k}$ are nonnegative, there holds:

Theorem 1 For every $P C B, H / U \leq R / U \leq 2$. Moreover, $H / U$ and $R / U$ can be made arbitrarily close to 2 for some PCBs.

Proof. Let us show that $H / U \leq R / U \leq 2$ for every PCB. First, notice that $H \leq R$, since the makespans $H$ and $R$ pertain to the same retrieval plan, and since the machine is clearly more efficient in unrestricted mode than in restricted mode. Therefore, we only need to prove that $R / U \leq 2$. Given an arbitrary PCB, consider the retrieval plan $S$ that achieves the optimal makespan $U$ on the unrestricted machine. Assume that $S$ calls for placing components $1, \ldots, n$ in this order, and for retrieving component $i$ from slot $j(i)(i=1, \ldots, n$ and $j(i) \in Y(i))$. Denote by $M$ the makespan of $S$ on the restricted machine. Then, we obtain successively:

$$
\begin{aligned}
R & \leq M \\
& =\sum_{i=1}^{n} \max \left\{\Delta g_{i}+\Delta c_{i}, \Delta g_{i}+\Delta m_{j(i) j(i+1)}, \Delta p_{i}+\Delta c_{i}, \Delta p_{i}+\Delta t_{i}\right\} \\
& \leq \sum_{i=1}^{n} \max \left\{\Delta g_{i}+\Delta c_{i}, \Delta g_{i}+\Delta m_{j(i) j(i+1)}\right\}+\sum_{i=1}^{n} \max \left\{\Delta p_{i}+\Delta c_{i}, \Delta p_{i}+\Delta t_{i}\right\} \\
& \leq 2 U .
\end{aligned}
$$

Indeed, inequality (4) holds by optimality of $R$ for the restricted machine, equality (5) follows from (3), and inequality (6)is trivial. As for inequality (7), observe that each sum in the left-hand side of (6) represents a lower bound on $U$, since each of them accounts for a sequence of operations - grip and place operations respectively - that must necessarily be performed in succession. Thus, we have established the first part of the theorem.

We now provide a small example showing that $H / U$ and $R / U$ can be made arbitrarily close to 2 . For the sake of simplicity, we assume in this example that the carousel of the machine only features two working heads and that it takes $|i-j|$ time units for the magazine rack to move from slot $i$ to slot $j$, for every pair of slots $i$ and $j$. (It would be an easy matter to extend this example so as to account for the more complex features of real machines). There are three components, say 1,2 and 3 , to be placed in this order. Component 1 is contained in slot $2 K$ of the magazine rack, component 2 is in slots $(K+1)$ and $3 K$, and component 3 is in slots 1 and $(3 K+1)$, where $K$ is a given integer. Moreover, the worktable requires $K$ time units to move from placement location 1 to placement location 2, and 1 time unit to move from location 2 to location 3. Each grip or place activity, and each rotation of the carousel, requires 1 time unit. 
With these data, it is easy to check that the optimal retrieval plan $S$ for the machine in unrestricted mode requires to grip component 2 from slot $3 K$ and component 3 from slot $(3 K+1)$. This plan entails the following sequence of operations (operations listed on a same line are performed concurrently during the timespan indicated):

1. grip component 1 in slot $2 K$ ( 1 time unit)

2. rotate the carousel, start moving the rack from slot $2 K$ to slot $3 K$ (for 1 time unit)

3. place component 1 , keep moving the rack towards slot $3 K$ (for 1 time unit)

4. move the rack to slot $3 K$, start moving the table from location 1 to location 2 (for $(K-2)$ time units)

5. grip component 2 from slot $3 K$, keep moving the table towards location 2 (for 1 time unit)

6. rotate the carousel, move the rack from slot $3 K$ to slot $(3 K+1)$, move the table to location 2 (1 time unit)

7. grip component 3 from slot $(3 K+1)$, place component 2 (1 time unit)

8. rotate the carousel, move the table from location 2 to location 3 (1 time unit)

9. place component 3 (1 time unit).

This sequence results in a makespan $U=(K+6)$.

One would similarly verify that, for the machine in restricted mode, the optimal retrieval plan $S^{\text {res }}$ consists in retrieving component 2 from slot $(K+1)$ and component 3 from slot 1 . The corresponding optimal makespan in restricted mode is equal to $R=(2 K+4)$, and is identical to the makespan of $S^{r e s}$ in unrestricted mode, i.e. $H=(2 K+4)$. Therefore, when $K$ goes to infinity, both $R / U$ and $H / U$ approach 2 as required.

Interestingly, it is also possible to prove that the makespan of the selection obtained by computing a shortest path in the grip graph (see end of Section 3) comes within a factor of 2 of the optimal CRP makespan, and that this bound is tight. We omit the proof of this result.

In conclusion, all the above comments underscore the need for an efficient and exact algorithm that takes into account all characteristic features of the Component Retrieval Problem. Such an algorithm will be proposed in the next section.

\section{A polynomial algorithm for CRP}

In this section, we consider a given CRP graph $D$, and we present a polynomial algorithm for CRP as formulated in Definition 4. As is explained in Section 3, the optimal selection in $D$ can generally not be computed by solving for a shortest path in the subgraph that is induced by the grip nodes of $D$, since the side-constraints that are induced by the precedence relations of the interfering place 
activities would be completely ignored in that case. The general approach in this section is to model each of these side-constraints as an arc between two grip nodes, with its length equal to the smallest longest path in $D$ between these grip nodes. The optimal selection can then be retrieved by solving for the shortest path in this newly constructed graph, which will be denoted by $D_{\mathcal{N}}$. Since the arc lengths in $D_{\mathcal{N}}$ can be computed by a (polynomial and simple) forward dynamic programming approach, and since a shortest path in $D_{\mathcal{N}}$ can be computed likewise, our procedure can be thought of as a "two-phase" forward dynamic programming algorithm.

This section is built up as follows. First, a simplified version of CRP will be considered, which can be solved by forward dynamic programming in polynomial time. The insights that have thus been obtained will then be used to arrive at a polynomial algorithm for CRP itself. Application of the proposed algorithm to the numerical example of Section 4 will conclude this section.

\subsection{A simplified version of CRP}

Lemma 1 The length of the path $\left(p_{0}, p_{1}, \ldots, p_{n+1}\right)$ through the place nodes is a lowerbound on the optimal solution value of $C R P$.

Proof. Straightforward.

This simple observation motivates our interest in the following problem.

\section{$\mathrm{CRP}^{*}$}

INPUT: A CRP graph $D$;

QUESTION: Is there a selection $S$ such that the path $\left(p_{0}, p_{1}, \ldots, p_{n+1}\right)$ through the place nodes is a longest path of $D(S)$ ?

Note that, if the answer to $\mathrm{CRP}^{*}$ is affirmative for some selection $S$, then $S$ is an optimal solution to CRP (cf. Lemma 1). For $0 \leq i \leq j \leq n+1$, let $L_{P}(i, j)$ be the length of the path $\left(p_{i}, p_{i+1}, \ldots, p_{j}\right)$ from $p_{i}$ to $p_{j}$ through the place nodes. Similarly, for a selection $S=\left\{g_{1}, g_{2}, \ldots, g_{n}\right\}$ and for $j-i \geq 2$, let $L_{G}(S, i, j)$ be the length of the path $\left(p_{i}, g_{i+1}, \ldots, g_{j-1}, p_{j}\right)$ from $p_{i}$ to $p_{j}$ with all intermediates nodes in $S$.

Theorem 2 For every selection $S$, the path $\left(p_{0}, p_{1}, \ldots, p_{n+1}\right)$ is a longest path of $D(S)$ if and only if $L_{G}(S, i, j) \leq L_{P}(i, j)$ for all $i, j \in\{0, \ldots, n+1\}$ with $j-i \geq 2$.

Proof. If $L_{G}(S, i, j)>L_{P}(i, j)$ for some $i, j$, then the path $\left(p_{0}, p_{1}, \ldots, p_{i}, g_{i+1}, \ldots, g_{j-1}, p_{j}, \ldots, p_{n+1}\right)$ is longer than the path through the place nodes. On the other hand, the inequalities in the theorem imply that the path through the place nodes will be at least as long as any path containing some grip nodes.

Theorem 2 motivates the introduction of a collection of s-labels associated with each selection $S$, which reflect the slack that $S$ displays with respect to the necessary and sufficient conditions stated in the theorem. 
Definition 5 For every selection $S$ and every $j \in\{1,2, \ldots, n\}$, define

$$
s(S, j)=\min _{0 \leq i \leq j-1}\left\{L_{P}(i, j+1)-L_{G}(S, i, j+1)\right\} .
$$

We view label $s(S, j)$ as being attached to the $j$-th grip node of $S$. Theorem 2 can now be equivalently stated as follows.

Corollary 1 For every selection $S$, the path $\left(p_{0}, \ldots, p_{n+1}\right)$ is a longest path of $D(S)$ if and only if

$$
s(S, j) \geq 0 \quad \text { for all } j \in\{1,2, \ldots, n\} .
$$

The $s$-labels satisfy the following recursion:

Lemma 2 For every selection $S=\left\{g_{1}, g_{2}, \ldots, g_{n}\right\}$ and every $j \in\{2,3, \ldots, n\}$,

$$
\begin{gathered}
s(S, j)=\min \left\{s(S, j-1)+L_{P}(j, j+1)+d\left(g_{j-1}, p_{j}\right)-d\left(g_{j-1}, g_{j}\right)-d\left(g_{j}, p_{j+1}\right),\right. \\
\left.L_{P}(j-1, j+1)-d\left(p_{j-1}, g_{j}\right)-d\left(g_{j}, p_{j+1}\right)\right\} .
\end{gathered}
$$

Proof. For each $i \in\{0, \ldots, j-2\}$, we can rewrite

$$
\begin{aligned}
& L_{P}(i, j+1)-L_{G}(S, i, j+1) \\
& \quad=\left[L_{P}(i, j)+L_{P}(j, j+1)\right]-\left[L_{G}(S, i, j)-d\left(g_{j-1}, p_{j}\right)+d\left(g_{j-1}, g_{j}\right)+d\left(g_{j}, p_{j+1}\right)\right] .
\end{aligned}
$$

The validity of the lemma follows directly from this observation and from Definition 5 .

Lemma 2 provides a recursive formulation of the $s$-labels associated for a given selection $S$. In order to solve $\mathrm{CRP}^{*}$, we now generalize the $s$-labels by introducing a label $s^{*}\left(g_{j}\right)$ attached to each grip node $g_{j} \in G_{j}$. The value of $s^{*}\left(g_{j}\right)$ is the largest value of $s(S, j)$ that can be attained by any selection $S$ containing $g_{j}$ and satisfying condition (9) up to layer $j-1$. More precisely,

Definition 6 For all $j \in\{1,2, \ldots, n\}$ and all $g_{j} \in G_{j}$, let $T\left(g_{j}\right)$ denote the set of selections $S$ with

(i). $g_{j} \in S$, and

(ii). $s(S, i) \geq 0$ for all $i \in\{1, \ldots, j-1\}$.

Then we define $s^{*}\left(g_{j}\right)=\max _{S \in T\left(g_{j}\right)} s(S, j)$.

As usual, we let $s^{*}\left(g_{j}\right)=-\infty$ when $T\left(g_{j}\right)=\emptyset$. Let us stress the following properties of the $s^{*}$-labels, which are direct consequences of Definition 6 . 
P1. $-\infty<s^{*}\left(g_{j}\right)<0$ if and only if $T\left(g_{j}\right) \neq \emptyset$ and $s(S, j)<0$ for every selection $S \in T\left(g_{j}\right)$.

P2. $s^{*}\left(g_{j}\right) \geq 0$ if and only if there exists a selection $S$ with $g_{j} \in S$ and $s(S, i) \geq 0$ for all $i \in\{1, \ldots, j\}$.

In particular, combining these properties with Corollary 1 renders

Theorem 3 The answer to $C R P^{\star}$ is affirmative if and only if $s^{*}\left(g_{n}\right) \geq 0$ for some node $g_{n} \in G_{n}$.

Similar to the $s$-labels, the $s^{*}$-labels can also be computed by dynamic programming (cf. Lemma 2).

Theorem 4 For all $j \in\{2,3, \ldots, n\}$ and for all $g_{j} \in G_{j}$,

$$
\begin{gathered}
s^{*}\left(g_{j}\right)= \\
\max _{g_{j-1} \in G_{j-1}, s^{*}\left(g_{j-1}\right) \geq 0} \min \left\{\quad s^{*}\left(g_{j-1}\right)+L_{P}(j, j+1)+d\left(g_{j-1}, p_{j}\right)-d\left(g_{j-1}, g_{j}\right)-d\left(g_{j}, p_{j+1}\right),\right. \\
\left.L_{P}(j-1, j+1)-d\left(p_{j-1}, g_{j}\right)-d\left(g_{j}, p_{j+1}\right)\right\} .
\end{gathered}
$$

Proof. Fix $j \in\{2,3, \ldots, n\}$ and $g_{j} \in G_{j}$. Denote the right-hand side of (10) by $\sigma$.

(i). Assume first that $T\left(g_{j}\right) \neq \emptyset$. Then, by Definition 6 , there exists an $S \in T\left(g_{j}\right)$ with $s^{*}\left(g_{j}\right)=$ $s(S, j)$. If we write $S=\left\{g_{1}, g_{2}, \ldots, g_{n}\right\}$, then it is clear that $S \in T\left(g_{j-1}\right)$, so $s(S, j-1) \leq$ $s^{*}\left(g_{j-1}\right)$. In addition, $s(S, j-1) \geq 0$. Combining the latter two inequalities with Lemma 2 , renders $-\infty<s^{*}\left(g_{j}\right)=s(S, j) \leq \sigma$.

(ii). Conversely, assume now that $\sigma>-\infty$, and let $g_{j-1} \in G_{j-1}$ attain the maximum in the definition of $\sigma$, i.e. $\sigma=\min \{a, b\}$ with $a=s^{*}\left(g_{j-1}\right)+L_{P}(j, j+1)+d\left(g_{j-1}, p_{j}\right)-d\left(g_{j-1}, g_{j}\right)-d\left(g_{j}, p_{j+1}\right)$ and $b=L_{P}(j-1, j+1)-d\left(p_{j-1}, g_{j}\right)-d\left(g_{j}, p_{j+1}\right)$. By Definition 6 , there exists a selection $S \in T\left(g_{j-1}\right)$ with $s^{*}\left(g_{j-1}\right)=s(S, j-1)$. Without loss of generality, we can assume that $g_{j} \in S$ (otherwise, substitute $g_{j}$ for the $j$-th grip node of $S$ ). Then, by Lemma $2, s(S, j)=\sigma$. On the other hand, since $s^{*}\left(g_{j-1}\right) \geq 0$, we deduce that $S \in T\left(g_{j}\right)$ and, by Definition 6 ,

$$
\sigma=s(S, j) \leq \max _{R \in T\left(g_{j}\right)} s(R, j)=s^{*}\left(g_{j}\right)
$$

Taken together, (i) and (ii) establish the theorem.

Theorem 4 implies that the $s^{*}$-labels can be computed in polynomial time, layer by layer. In view of Theorem 3, we have thus obtained a polynomial algorithm for the solution of CRP*. This algorithm can be implemented to run in $O(e)$ time, where $e$ is the number of arcs of $D$. Moreover, the proof of Theorem 4 establishes that, in addition to answering $\mathrm{CRP}^{*}$, we can also find a selection $S$ with $s(S, j) \geq 0$ for all $0 \leq j \leq n$ if one exists. As a final remark, we observe that, up to this point, we have not made any use of the properties of arc lengths recorded in Definition 1. In other words, Theorem 4 applies for arbitrary arc lengths. 


\subsection{Further properties of the $s$-labels}

We have just described the role that the $s$-labels play in solving CRP*. In the next subsection, these ideas will be incorporated into an algorithm for the full-fledged Component Retrieval Problem. In order to achieve this goal, we first need to understand some of the basic properties of the $s$-labels. These properties will now be recorded in a sequence of lemmas.

Lemma 3 For any $j \in\{1,2, \ldots, n\}$ and $g_{j} \in G_{j}$, let $S=\left\{g_{1}, g_{2}, \ldots, g_{n}\right\}$ be a selection in $T\left(g_{j}\right)$. Consider $D(S)$. Then

(i). the path $\left(p_{0}, p_{1}, \ldots, p_{j}\right)$ is a longest path from $p_{0}$ to $p_{j}$ with length $L_{P}(0, j)$;

(ii). the path $\left(p_{0}, p_{1}, \ldots, p_{i}, g_{i+1}, g_{i+2}, \ldots, g_{j}\right)$ is a longest path from $p_{0}$ to $g_{j}$ with length

$$
L_{P}(0, j+1)-s(S, j)-d\left(g_{j}, p_{j+1}\right)
$$

where $i$ is any index that realizes the minimum in the expression (8) defining $s(S, j)$.

\section{Proof.}

(i). By Definition $6, s(S, i) \geq 0$ for all $i \in\{1, \ldots, j-1\}$. The claim is now a straighforward extension of Corollary 1.

(ii). Let $P_{g_{j}}$ be any longest path from $p_{0}$ to $g_{j}$ and let $k=\max \left\{\ell \mid p_{\ell} \in P_{g_{j}}\right\}$. Since $0 \leq k \leq j-1$, we have $S \in T\left(g_{k}\right)$, and hence $\left(p_{0}, p_{1}, \ldots, p_{k}\right)$ is a longest path from $p_{0}$ to $p_{k}$ (cf. (i)). Thus, without loss of generality, we can assume that $P_{g_{j}}=\left(p_{0}, p_{1}, \ldots, p_{k}, g_{k+1}, \ldots, g_{j}\right)$. The length of $P_{g_{j}}$ is now easily checked to be given by

$$
L_{P}(0, j+1)-\left(L_{P}(k, j+1)-L_{G}(S, k, j+1)\right)-d\left(g_{j}, p_{j+1}\right) .
$$

In view of (8), the latter expression is maximized when $L_{P}(k, j+1)-L_{G}(S, k, j+1)=s(S, j)$, i.e. when $k=i$. Thus we may indeed conclude that the path $\left(p_{0}, p_{1}, \ldots, p_{i}, g_{i+1}, g_{i+2}, \ldots, g_{j}\right)$ is a longest path from $p_{0}$ to $g_{j}$ with length as stated in (11).

Next, let us consider what happens when, in a selection induced subgraph $D(S)$, the longest path is not the path of place nodes $\left(p_{0}, p_{1}, \ldots, p_{n+1}\right)$ (this is the only interesting case, since we know from the previous subsection how to handle yes-instances of $\mathrm{CRP}^{*}$ ). In such a case, we already know by Corollary 1 that $s(S, j)$ must be negative for some layer $j$. Let us consider the first such layer.

Lemma 4 For any selection $S=\left\{g_{1}, g_{2}, \ldots, g_{n}\right\}$, let $j$ be the smallest index in $\{1,2, \ldots, n\}$ with $s(S, j)<0$. Then, in $D(S)$, every longest path from $p_{0}$ to $p_{n+1}$ contains $g_{j}$.

Proof. Since every path from $p_{0}$ to $p_{n+1}$ goes through either $p_{j+1}$ or $g_{j+1}$, it suffices to show that $g_{j}$ is contained in every longest path from $p_{0}$ to $p_{j+1}$ and from $p_{0}$ to $g_{j+1}$. Since $S \in T\left(g_{j}\right)$ (by definition 
of $j$ ), the length of a longest path from $p_{0}$ to $p_{j}$ (resp. $g_{j}$ ) is given by Lemma 3 . Thus, it suffices to show that

$$
\left(L_{P}(0, j+1)-s(S, j)-d\left(g_{j}, p_{j+1}\right)\right)+d\left(g_{j}, p_{j+1}\right)>L_{P}(0, j)+d\left(p_{j}, p_{j+1}\right)
$$

(i.e., the longest path to $p_{j+1}$ via $g_{j}$ is longer than the longest path to $p_{j+1}$ via $p_{j}$ ), and that

$$
\left(L_{P}(0, j+1)-s(S, j)-d\left(g_{j}, p_{j+1}\right)\right)+d\left(g_{j}, g_{j+1}\right)>L_{P}(0, j)+d\left(p_{j}, g_{j+1}\right)
$$

(i.e., the longest path to $g_{j+1}$ via $g_{j}$ is longer than the longest path to $g_{j+1}$ via $g_{j}$ ).

Now, (12) is trivially equivalent to the assumption that $s(S, j)<0$. On the other hand, according to Definition 1,

$$
d\left(g_{j}, g_{j+1}\right)+d\left(p_{j}, p_{j+1}\right) \geq d\left(g_{j}, p_{j+1}\right)+d\left(p_{j}, g_{j+1}\right) .
$$

The inequality (13) is then obtained by the addition of (14) to (12).

For an arbitrary selection $S=\left\{g_{1}, g_{2}, \ldots, g_{n}\right\}$, Lemma 4 suggests that a longest path of $D(S)$ can be obtained by the following procedure. (Let us mention right away that this procedure is much more involved than necessary, if its only purpose is to obtain a longest path of $D(S)$. The reason for considering it in this form is that it will rather naturally lead to an algorithm for CRP.) First, compute all labels $s(S, j)$ (e.g. layer by layer, as suggested by Lemma 2). If all $s$-labels are nonnegative, then we know that $\left(p_{0}, p_{1}, \ldots, p_{n+1}\right)$ is a longest path of $D(S)$. Otherwise, let

$$
j=\min \{k \in\{1, \ldots, n\} \mid s(S, k)<0\} .
$$

In view of Lemma 4 , a longest path from $p_{0}$ to $p_{n+1}$ in $D(S)$ can be obtained by concatenating a longest path from $p_{0}$ to $g_{j}$ with a longest path from $g_{j}$ to $p_{n+1}$. Accordingly, for any selection $S$, we call the first grip node $g_{j} \in S$ for which $s(S, j)$ is negative, a reset node of $D(S)$. The terminology reset expresses the fact that the computation of a longest path of $D(S)$ can be started anew from such a node. Now, by Lemma 3 , a longest path from $p_{0}$ to $g_{j}$ is readily available. Thus, we only need to find a longest path from $g_{j}$ to $p_{n+1}$ in $D(S)$. This subproblem clearly has the same structure as the problem we started with. More precisely, we can handle it as follows. We discard from $D(S)$ all layers with index $i \leq j$, except for $g_{j}$. Moreover, we decrease the length of both arcs $\left(g_{j}, g_{j+1}\right)$ and $\left(g_{j}, p_{j+1}\right)$ by $d\left(g_{j}, p_{j+1}\right)$ (this is to account for the last term of (11); see (15) hereunder). Denote the new CRP graph thus constructed by $D_{g_{j}}(S)$. The observation we make now is that, as a consequence of Lemma 3 and Lemma 4,

$$
L(D(S))=L_{P}(0, j+1)-s(S, j)+L\left(D_{g_{j}}(S)\right)
$$

(cf. Definition 3). The procedure just described can be applied iteratively until either $g_{n}$ receives a nonnegative label or $g_{n}$ becomes a reset node. In either case, let $u_{1}, u_{2}, \ldots, u_{r}$ denote the reset nodes 
sequentially identified in the process. Thus, for $k=1, \ldots, r, u_{k}$ is the reset node of $D_{u_{k-1}}(S)$ (where we let $\left.u_{0} \equiv p_{0}\right)$. Denote by $s\left(S, u_{k-1}, u_{k}\right)$ the (negative) $s$-label attached to $u_{k}$ in $D_{u_{k-1}}(S)$. The previous discussion can then be summarized as follows.

Lemma 5 If $u_{k}$ is the reset node of $D_{u_{k-1}}(S)$ for $k=1, \ldots, r$, and $D_{u_{r}}(S)$ has no reset node, then

$$
L(D(S))=L_{P}(0, n+1)-\sum_{k=1}^{r} s\left(S, u_{k-1}, u_{k}\right)
$$

Proof. This statement is a consequence of Lemma 3 and Lemma 4, and the foregoing discussion. More precisely, let $u_{r}$ lie in $G_{\ell}$, where $1 \leq \ell \leq n$. Then, induction on (15) leads to

$$
L(D(S))=L_{P}(0, \ell+1)-\sum_{k=1}^{r} s\left(S, u_{k-1}, u_{k}\right)+L\left(D_{u_{r}}(S)\right) .
$$

There are now two cases. If $\ell=n$, i.e. the reset node $u_{r}$ coincides with $g_{n} \in S$, then $L\left(D_{u_{r}}(S)\right)=0$, from which (16) follows. Conversely, if $g_{n}$ is not a reset node, then $L\left(D_{u_{r}}(S)\right)=L_{P}(\ell+1, n+1)$ (by Corollary 1), and (16) follows again.

Finally, consider two selections in $T\left(g_{j}\right)$, which are identical from layer $j$ onwards, but which have different $s$-label values at layer $j$. The next lemma states sufficient conditions for one of the selections to dominate the other one, as far as minimizing $L(D(S))$ is concerned.

Lemma 6 For any $j \in\{1,2, \ldots, n\}$ and $g_{j} \in G_{j}$, let $S=\left\{g_{1}, g_{2}, \ldots, g_{n}\right\}$ and $S^{\prime}=\left\{g_{1}^{\prime}, g_{2}^{\prime}, \ldots, g_{n}^{\prime}\right\}$ be two selections in $T\left(g_{j}\right)$ with $g_{i}=g_{i}^{\prime}$ for $i=j, \ldots, n$. If $s(S, j)<s\left(S^{\prime}, j\right)$ and $s(S, j)<0$, then $L\left(D\left(S^{\prime}\right)\right)<L(D(S))$.

Proof. Let $P$ be any longest path in $D\left(S^{\prime}\right)$. Then, $P$ contains either $p_{j}$ or $g_{j}$. In the first case, we can assume without loss of generality that $P$ contains $p_{0}, \ldots, p_{j}$ (cf. Lemma 3 sub (i)), so that $P$ is also a path in $D(S)$. But then Lemma 4 implies that $P$ is not a longest path of $D(S)$, hence $L\left(D\left(S^{\prime}\right)\right)<L(D(S))$. Conversely, if $P$ contains $g_{j}$, then Lemma 3 sub (ii) states that the subpath of $P$ from $p_{0}$ to $g_{j}$ has length $L_{P}(0, j+1)-s\left(S^{\prime}, j\right)-d\left(g_{j}, p_{j+1}\right)$, and that the longest path from $p_{0}$ to $g_{j}$ in $D(S)$ has length $L_{P}(0, j+1)-s(S, j)-d\left(g_{j}, p_{j+1}\right)$. Since the latter is strictly larger than the former, the result follows.

\subsection{The general case}

Below we are going to show how Lemmas 5 and 6 can be combined to produce a polynomial time algorithm for CRP. First, we reformulate and extend some of the notation introduced earlier. For every node $g_{j} \in G_{j}$ of $D(j=0,1, \ldots, n-1)$, we denote by $D_{g_{j}}$ the subgraph of $D$ induced by the node set $\left\{g_{j}\right\} \cup \bigcup_{i=j+1}^{n+1} L_{i}$. The arc lengths in $D_{g_{j}}$ are the same as in $D$, except that the length of each arc leaving $g_{j}$ is decreased by $d\left(g_{j}, p_{j+1}\right)$ for all $j \geq 1$. Note that $D_{g_{0}}$ is thus identical to $D$. For each graph $D_{g_{j}}$, we can define $s^{*}$-labels as we did for graph $D$ in Definition 6 ; the $s^{*}$-label attached to node $g_{k}$ in $D_{g_{j}}$ is denoted by $s^{*}\left(g_{j}, g_{k}\right)\left(g_{k} \in \bigcup_{i=j+1}^{n} G_{i}\right)$. In addition, $S_{g_{j} g_{k}}$ will refer to any selection that realizes the value of $s^{*}\left(g_{j}, g_{k}\right)$. 
Before giving a more formal description of our algorithm, let us clarify the intuition behind it. Observe that, according to (16), CRP can be seen as the problem of minimizing the expression $\sum_{k}-s\left(S, u_{k-1}, u_{k}\right)$ over all possible selections $S$. Let $S$ be an optimal selection of the CRP graph $D$, and let $u_{1}, \ldots, u_{r}$ be the corresponding sequence of reset nodes. By definition of the quantities $s\left(S, u_{k-1}, u_{k}\right)$, we have $s\left(S, u_{0}, u_{1}\right)=s(S, j)$ if $u_{1}$ is in layer $j$. Consider now the selection $S_{u_{0} u_{1}}$, which is such that (by definition)

$$
s\left(S_{u_{0} u_{1}}, j\right)=s^{*}\left(u_{0}, u_{1}\right)=\max _{R \in T\left(u_{1}\right)} s(R, j),
$$

and suppose that

$$
s(S, j)<s\left(S_{u_{0} u_{1}}, j\right)
$$

Then, construct the selection $S^{\prime}$ that coincides with $S_{u_{0} u_{1}}$ from layer 1 to layer $j$ and that coincides with $S$ from layer $j$ to layer $n$. It follows directly from Lemma 6 that $S^{\prime}$ dominates $S$, and this contradicts the optimality of $S$. Thus we have established that

$$
s(S, j)=s^{*}\left(u_{0}, u_{1}\right),
$$

or equivalently

$$
s\left(S, u_{0}, u_{1}\right)=s^{*}\left(u_{0}, u_{1}\right) .
$$

By repeating this argument $r$ times, we can derive that

$$
-\sum_{k=1}^{r} s\left(S, u_{k-1}, u_{k}\right)=-\sum_{k=1}^{r} s^{*}\left(u_{k-1}, u_{k}\right)
$$

In this way, we have reduced CRP to the problem of minimizing the right-hand side of (17) over all possible choices of the $u_{k}$ 's, under the restriction that these nodes are the sequence of reset nodes associated with some selection.

We will now translate the latter problem into a shortest path problem in an auxiliary network $D_{\mathcal{N}}$, where the length of each arc $\left(g_{j}, g_{k}\right)$ is "essentially" equal to $-s^{*}\left(g_{j}, g_{k}\right)$. More precisely, the node set of $D_{\mathcal{N}}$ is $\left\{g_{0}\right\} \cup \bigcup_{i=1}^{n} G_{i}$. The arcs of $D_{\mathcal{N}}$ are all pairs of nodes of the form $\left(g_{j}, g_{k}\right)$ where $g_{j} \in G_{j}$, $g_{k} \in G_{k}$ and $0 \leq j<k \leq n$. The length of arc $\left(g_{j}, g_{k}\right)$ is defined to be $w\left(g_{j}, g_{k}\right)$, where

Case 1: $w\left(g_{j}, g_{k}\right)=-s^{*}\left(g_{j}, g_{k}\right)$ if $-\infty<s^{*}\left(g_{j}, g_{k}\right)<0$;

Case 2: $w\left(g_{j}, g_{k}\right)=0$ if $s^{*}\left(g_{j}, g_{k}\right) \geq 0$ and $k=n$;

Case 3: $w\left(g_{j}, g_{k}\right)=\infty$ otherwise.

In view of Definition 6 and Theorem 4, Case 1 corresponds to a situation where $g_{k}$ is a reset node in the subgraph of $D_{g_{j}}$ induced by the selection $S_{g_{j} g_{k}}$ (see property P1 following Definition 6 ). Similarly, 
Case 2 occurs when there is no reset node in the subgraph induced by $S_{g_{j} g_{n}}$ up to and including layer $n$. Finally, in Case 3, any reset node of the subgraph induced by $S_{g_{j} g_{k}}$ lies either before $g_{k}$ $\left(s^{*}\left(g_{j}, g_{k}\right)=-\infty\right)$ or after $g_{k}\left(s^{*}\left(g_{j}, g_{k}\right) \geq 0\right.$ and $\left.k<n\right)$.

Denote by $w(P)$ the length of a path $P$ in $D_{\mathcal{N}}$. For brevity, when we write "shortest path in $D_{\mathcal{N}}$ ", we mean "shortest path in $D_{\mathcal{N}}$ from $g_{0}$ to some node in $G_{n}$, with respect to the length function $w$ ". We are now finally ready for our next, and main, result. Note, however, that its proof is simply a formal generalization of the arguments presented above.

Theorem 5 The length of a shortest path in $D_{\mathcal{N}}$ is equal to $L_{P}(0, n+1)+L(D)$, where $L(D)$ is the optimal value of the Component Retrieval Problem on the graph D.

\section{Proof.}

(i). Let $S$ be an optimal selection for $C R P$ and let $u_{k}$ denote the reset node of $D_{u_{k-1}}(S)$ $\left(k=1, \ldots, r ; u_{0}=g_{0}\right)$. By Lemma 5 , equation (16) holds. Let now $P^{\prime}=\left\{u_{0}, u_{1}, \ldots, u_{r}\right\}$, and consider any index $k \in\{1, \ldots, r\}$. By definition of reset nodes, $s\left(S, u_{k-1}, u_{k}\right)<0$ and $s\left(S, u_{k-1}, u\right) \geq 0$ for all grip nodes $u$ lying between $u_{k-1}$ and $u_{k}$ in $S$. Therefore, by Definition 6 , we get $S \in T\left(u_{k}\right)$, where $T\left(u_{k}\right)$ is defined with respect to the graph $D_{u_{k-1}}$, and this implies that $-\infty<s\left(S, u_{k-1}, u_{k}\right) \leq \min \left\{0, s^{*}\left(u_{k-1}, u_{k}\right)\right\}$. If, for all $k=1, \ldots, r, w\left(u_{k-1}, u_{k}\right)$ is defined by either Case 1 or Case 2 (cf. (18)-(19)), then,

$$
-s\left(S, u_{k-1}, u_{k}\right) \geq w\left(u_{k-1}, u_{k}\right) \text { for } k=1, \ldots, r .
$$

Combining these inequalities with (16) yields

$$
L(D) \geq L_{P}(0, n+1)+w\left(P^{\prime}\right) .
$$

Thus, assume now that $w\left(u_{k-1}, u_{k}\right)$ is defined by Case 3 (see (20)) for some $k$. In that case, $s^{*}\left(u_{k-1}, u_{k}\right) \geq 0$, or equivalently $s\left(S_{u_{k-1} u_{k}}, u_{k-1}, u_{k}\right) \geq 0$ (notice that $s^{*}\left(u_{k-1}, u_{k}\right)=-\infty$ has been ruled out earlier). Let now $S^{\prime}$ denote the selection which coincides with $S$ from $p_{0}$ to $u_{k-1}$ and from $u_{k}$ to $p_{n+1}$, and which coincides with $S_{u_{k-1} u_{k}}$ from $u_{k-1}$ to $u_{k}$. Apply Lemma 6 to the selections $S$ and $S^{\prime}$, both viewed as selections of $D_{u_{k-1}}$. This lemma implies that the longest path in the subgraph of $D_{u_{k-1}}$ induced by $S^{\prime}$ is shorter than the longest path in the subgraph induced by $S$, contradicting the optimality of $S$. As a result, the case that $w\left(u_{k-1}, u_{k}\right)$ is defined by $(20)$ does not occur for arcs $\left(u_{k-1}, u_{k}\right)$ on paths in $D_{\mathcal{N}}$ that are defined by the reset nodes of optimal selections.

(ii). Conversely, let $P=\left\{u_{0}, u_{1}, \ldots, u_{r}\right\}$ be a shortest path in $D_{\mathcal{N}}$, with $u_{0}=g_{0}$ and $u_{r} \in G_{n}$. From (i) it follows that $w(P) \leq w\left(P^{\prime}\right)<\infty$. Hence, for $k=1, \ldots, r-1$, the $w\left(u_{k-1}, u_{k}\right)$ 's on $P$ are all defined by (18), which means that $u_{k}$ is a reset node in the subgraph of $D_{u_{k-1}}$ induced by the selection $S_{u_{k-1} u_{k}}$. Now consider the selection $S=\cup_{1 \leq k \leq r} S_{u_{k-1} u_{k}}^{\prime}$, where $S_{u_{k-1} u_{k}}^{\prime}$ is the set of nodes of $S_{u_{k-1} u_{k}}$ that lie between $u_{k-1}$ and $u_{k}$. Lemma 5 implies that $L(D(S))=$ $L_{P}(0, n+1)+w(P)$, and hence

$$
L(D) \leq L_{P}(0, n+1)+w(P) .
$$

From (21) and (22), we conclude that $L(D) \leq L_{P}(0, n+1)+w(P) \leq L_{P}(0, n+1)+w\left(P^{\prime}\right) \leq L(D)$. This establishes the result. 
In summary, the Component Retrieval Problem can be solved by the following algorithm:

\section{procedure SOLVE-CRP:}

begin

for all $j=0,1, \ldots, n-1$ and for all $g_{j} \in G_{j}$ do

begin

set up the graph $D_{g_{j}}$;

for all $k=j+1, \ldots, n$ and all $g_{k} \in G_{k}$ do

begin

compute the label $s^{*}\left(g_{j}, g_{k}\right)$ of node $g_{k}$ in $D_{g_{j}}$, and the corresponding selection $S_{g_{j} g_{k}}$; define $w\left(g_{j}, g_{k}\right)$ according to (18), (19) and (20);

end

end

set up the graph $N$;

compute a shortest path in $D_{\mathcal{N}}$ from $g_{0}$ to $G_{n}$ with respect to the length function $w$;

let $P=\left\{u_{0}, u_{1}, \ldots, u_{r}\right\}$ denote this shortest path;

return the optimal selection $S=\cup_{1 \leq k \leq r} S_{u_{k-1} u_{k}}^{\prime}$ with length $L(D)=L_{P}(0, n+1)+w(P)$

end

Theorem 6 Procedure SOLVE-CRP is correct and solves the Component Retrieval Problem in $O(v e)$ time on a CRP graph $D$ with $v$ nodes and $e$ arcs.

Proof. The correctness of procedure SOLVE-CRP follows from (the proof of) Theorem 5 . As for its complexity, note that each execution of the loop "for all $j$, for all $g_{j}$ " requires $O(e)$ time (by the comments following Theorem 4), and that this loop is executed $O(v)$ times. A shortest path in $D_{\mathcal{N}}$ can be found in $O\left(v^{2}\right)$ time since $D_{\mathcal{N}}$ is acyclic (see e.g. Ahuja, Magnanti and Orlin (1993)).

The complexity of procedure SOLVE-CRP can be alternatively stated as follows. Let $m$ be an upper-bound on the number of feeders of each type, i.e. $m=\max _{1 \leq i \leq n}\left|G_{i}\right|$. Then, $v=O(m n)$ and $e=O\left(m^{2} n\right)$, so that SOLVE-CRP runs in $O\left(m^{3} n^{2}\right)$ time.

\subsection{Example}

Below we will illustrate the algorithm of the previous subsection by applying it to the problem instance that was described in Section 4, Figure 3, with $n=5$. Recall that $d\left(g_{4}, g_{5}\right)=4+x$ in this problem, with $x$ being equal to either 0 or 5 . The first phase yields the $s^{*}$-labels; the relevant values of these labels are listed in Table 4.

The auxiliary graph $D_{\mathcal{N}}$ has node set $\left\{g_{0}, g_{1}^{1}, g_{1}^{2}, g_{2}^{1}, g_{2}^{2}, g_{3}, g_{4}, g_{5}\right\}$; its relevant arcs are listed in Table 5 . If $x=0$, the shortest path of $D_{\mathcal{N}}$ is $\left(g_{0}, g_{3}, g_{5}\right)$ with a length of 2 . Tracing back the predecessors in the third column of Table 4 reveals the corresponding optimal selection $\left\{g_{1}^{1}, g_{2}^{1}, g_{3}, g_{4}, g_{5}\right\}$, which has 


\begin{tabular}{|c|c|c|}
\hline \hline grip node pair $\left(g_{i}, g_{j}\right)$ & $s^{*}\left(g_{i}, g_{j}\right)$ & arg max in $(10)$ \\
\hline$\left(g_{0}, g_{1}^{1}\right)$ & 2 & $g_{0}$ \\
$\left(g_{0}, g_{1}^{2}\right)$ & 2 & $g_{0}$ \\
$\left(g_{0}, g_{2}^{1}\right)$ & 2 & $g_{1}^{1}$ \\
$\left(g_{0}, g_{2}^{2}\right)$ & -4 & $g_{1}^{2}$ \\
$\left(g_{0}, g_{3}\right)$ & -2 & $g_{2}^{1}$ \\
$\left(g_{2}^{2}, g_{3}\right)$ & 3 & $g_{2}^{2}$ \\
$\left(g_{2}^{2}, g_{4}\right)$ & 3 & $g_{3}$ \\
$\left(g_{3}, g_{4}\right)$ & 0 & $g_{3}$ \\
$\left(g_{2}^{2}, g_{5}\right)$ & $3-x$ & $g_{4}$ \\
$\left(g_{3}, g_{5}\right)$ & $-x$ & $g_{4}$ \\
\hline \hline
\end{tabular}

Table 4: (Relevant) $s^{*}$-labels for the numerical example.

\begin{tabular}{|c|c|c|}
\hline \hline Arc & Length & Remark \\
\hline$\left(g_{0}, g_{2}^{2}\right)$ & 4 & \\
$\left(g_{0}, g_{3}\right)$ & 2 & \\
$\left(g_{2}^{2}, g_{5}\right)$ & 2 & only if $x=5$ \\
$\left(g_{3}, g_{5}\right)$ & 5 & only if $x=5$ \\
$\left(g_{2}^{2}, g_{5}\right)$ & 0 & only if $x=0$ \\
$\left(g_{3}, g_{5}\right)$ & 0 & only if $x=0$ \\
\hline \hline
\end{tabular}

Table 5: (Relevant part of) graph $D_{\mathcal{N}}$ for the numerical example.

a makespan of $16+2=18$ (cf. Theorem 5). On the other hand, if $x=5$, then the shortest path in $D_{\mathcal{N}}$ is $\left(g_{0}, g_{2}^{2}, g_{5}\right)$ with a length of 6 . The corresponding optimal selection reads $\left\{g_{1}^{2}, g_{2}^{2}, g_{3}, g_{4}, g_{5}\right\}$, which has a makespan of $16+6=22$. Note that these outcomes are consistent with the optimal selections that were reported in Section 4.

\section{An $N P$-hard generalization of CRP.}

Our definition of the Component Retrieval Problem includes condition (1) on the arc lengths of CRP graphs. This condition has been explicitly used in the proof of Lemma 5 in Section 5. In this section we will show that the problem becomes $N P$-hard when condition (1) is absent. Consider the following decision problem (Generalized $C R P$ ).

\section{GCRP}

INPUT: An integer $\beta$ and a graph $D$ satisfying the assumptions of Definition 1, except for (1). QUESTION: Is there a selection $S$ such that the longest path in the selection induced subgraph $D(S)$ has length at most $\beta$ ? 


\begin{tabular}{|l|l|l|}
\hline \hline \multicolumn{1}{|c|}{ Arc } & \multicolumn{1}{|c|}{ Length } & \multicolumn{1}{c|}{ For } \\
\hline$\left(g_{4 i-3}^{1}, g_{4 i-2}^{1}\right)$ & $K+x_{2 i-1}$ & $i=1, \ldots, N$ \\
$\left(g_{4 i-3}^{2}, g_{4 i-2}^{2}\right)$ & $K+x_{2 i}$ & $i=1, \ldots, N$ \\
$\left(g_{4 i-2}^{1}, g_{4 i-1}^{1}\right)$ & 0 & $i=1, \ldots, N$ \\
$\left(g_{4 i-2}^{2}, g_{4 i-1}^{2}\right)$ & 0 & $i=1, \ldots, N$ \\
$\left(g_{4 i-1}^{1}, g_{4 i}^{1}\right)$ & $K+x_{2 i}$ & $i=1, \ldots, N$ \\
$\left(g_{4 i-1}^{2}, g_{4 i}^{2}\right)$ & $K+x_{2 i-1}$ & $i=1, \ldots, N$ \\
$\left(g_{4 i}^{1}, g_{4 i+1}^{1}\right)$ & $K$ & $i=1, \ldots, N$ \\
$\left(g_{4 i}^{1}, g_{4 i+1}^{2}\right)$ & $K$ & $i=1, \ldots, N$ \\
$\left(g_{4 i}^{2}, g_{4 i+1}^{1}\right)$ & $K$ & $i=1, \ldots, N$ \\
$\left(g_{4 i}^{2}, g_{4 i+1}^{2}\right)$ & $K$ & $i=1, \ldots, N$ \\
$\left(g_{k}^{1}, g_{k+1}^{2}\right)$ & $M$ & $k \in\{4 i-3,4 i-2,4 i-1\}$ \\
$\left(g_{k}^{2}, g_{k+1}^{1}\right)$ & $M$ & $k \in\{4 i-3,4 i-2,4 i-1\}$ \\
$\left(p_{4 i-2}, p_{4 i-1}\right)$ & 0 & $i=1, \ldots, N$ \\
other place arcs & $K$ & \\
all cross arcs $^{3}$ & $Q$ & \\
\hline \hline
\end{tabular}

Table 6: Arc lengths of the GCRP instance $(D, \beta)$

Theorem 7 GCRP is $N P$-complete, even if $\left|G_{i}\right| \leq 2$ for $i=1, \ldots, n$.

Proof. GCRP is clearly in NP. Below we will present a polynomial transformation from the $N P$ complete Even-Odd Partitioning problem (EOP; see Garey and Johnson (1979)) to CRP.

EOP

INPUT: $N$ pairs of positive integers $I_{i}=\left\{x_{2 i-1}, x_{2 i}\right\}$ for $i=1, \ldots, N$.

QUESTION: Is there an even-odd partition of $\{1,2, \ldots, 2 N\}$, i.e. a partition of $\{1,2, \ldots, 2 N\}$ into disjoint subsets $A$ and $B$ with $\left|A \cap I_{i}\right|=\left|B \cap I_{i}\right|=1$ for $i=1, \ldots, N$, and $\sum_{i \in A} x_{i}=\sum_{i \in B} x_{i}$ ?

Given an instance of EOP, we define a graph $D$ as in Definition 1 , with $n=4 N$. For $k=1, \ldots, 4 N$, each layer $k$ contains three nodes, namely one place node $p_{k}$ and two grip nodes $g_{k}^{1}$ and $g_{k}^{2}$. Layers $4 i-3,4 i-2,4 i-1$ and $4 i$ are associated with pair $I_{i}$ in the instance of $\operatorname{EOP}(1 \leq i \leq N)$. In order to define the arc lengths, we introduce three large numbers of different magnitudes:

$$
\begin{aligned}
Q & =(N+1) \cdot \max _{1 \leq i \leq 2 N}\left\{x_{i}\right\} \\
K & =(N+1) Q \\
M & =4(N+1) K
\end{aligned}
$$

The arc lengths in $D$ are listed in Table 6 . (For notational convenience we indifferently denote the sink of $D$ by $p_{n+1}, g_{n+1}^{1}$ or $g_{n+1}^{2}$.) Recall that arcs emanating from $g_{0}$ have length zero. Finally, we set $\beta=N(3 K+Q)+\frac{1}{2} \sum_{i=1}^{2 N} x_{i}$. This completely specifies an instance $(D, \beta)$ of GCRP. It remains to show that the instance of GCRP obtained in this way and the original instance of EOP have the same answer.

\footnotetext{
${ }^{3}$ A place arc connects two place nodes.

${ }^{3} \mathrm{~A}$ cross arc connects a grip and a place node.
} 
(i). Suppose first that the instance of EOP has a positive answer, and let $(A, B)$ define an even-odd partition of $\{1,2, \ldots, 2 N\}$. Without loss of generality we may assume that $A=\{2 i-1 \mid i=$ $1, \ldots, N\}$ and $B=\{2 i \mid i=1, \ldots, N\}$. Consider the selection $S$ that contains $g_{4 i-3}^{1}, g_{4 i-2}^{1}, g_{4 i-1}^{1}$ and $g_{4 i}^{1}$ for $i$ is odd, and $g_{4 i-3}^{2}, g_{4 i-2}^{2}, g_{4 i-1}^{2}$ and $g_{4 i}^{2}$ for $i$ is even $(i=1, \ldots, N)$. We now claim that $L(D(S))=\beta$, implying that the instance $(D, \beta)$ has a positive answer.

Denote by $D_{i}$ the subgraph of $D(S)$ induced by layers $4 i-3,4 i-2, \ldots, 4 i+1$, for every $i \in\{1,2, \ldots, N\}$. Two candidate longest paths in $D_{i}$ are of the form

$$
\left\{p_{4 i-3}, p_{4 i-2}, g_{4 i-1}^{\delta}, g_{4 i}^{\delta}, g_{4 i+1}^{3-\delta}\right\} \text { and }\left\{g_{4 i-3}^{\delta}, g_{4 i-2}^{\delta}, p_{4 i-1}, p_{4 i}, p_{4 i+1}\right\} \text {, }
$$

respectively, where $\delta=1$ when $i$ is odd, and $\delta=2$ otherwise. One of these paths has length $3 K+Q+x_{2 i-1}$ and the other one has length $3 K+Q+x_{2 i}$. Furthermore, it is easily seen that all other paths in $D_{i}$ are strictly shorter than the ones in (23). Using mathematical induction to $N$ then reveals that any longest path from $g_{0}$ to $p_{4 N+1}$ in $D(S)$ is the concatenation of paths in $D_{i}$ of the types mentioned in $(23)(i=1,2, \ldots, N)$. Hence, the two candidate longest paths in $D(S)$ are

$$
\begin{aligned}
P_{1} & =\left(g_{0}, g_{1}^{1}, g_{2}^{1}, p_{3}, p_{4}, p_{5}, p_{6}, g_{7}^{2}, g_{8}^{2}, g_{9}^{1}, g_{10}^{1}, p_{11}, p_{12}, p_{13}, p_{14}, g_{15}^{2}, g_{16}^{2}, g_{17}^{1}, \ldots, p_{4 N+1}\right) \\
& =\left\{g_{0}\right\} \cup \underset{\substack{1 \leq i<N, i \text { odd }}}{\bigcup}\left\{g_{4 i-3}^{1}, g_{4 i-2}^{1}, p_{4 i-1}, p_{4 i}\right\} \cup \underset{\substack{1<i<N, i \text { even }}}{\bigcup}\left\{p_{4 i-3}, p_{4 i-2}, g_{4 i-1}^{2}, g_{4 i}^{2}\right\} \cup\left\{p_{4 N+1}\right\}
\end{aligned}
$$

and

$$
\begin{aligned}
P_{2} & =\left(g_{0}, p_{1}, p_{2}, g_{3}^{1}, g_{4}^{1}, g_{5}^{2}, g_{6}^{2}, p_{7}, p_{8}, p_{9}, p_{10}, g_{11}^{1}, g_{12}^{1}, g_{13}^{2}, g_{14}^{2}, p_{15}, p_{16}, p_{17}, \ldots, p_{4 N+1}\right) \\
& =\left\{g_{0}\right\} \cup \underset{\substack{1 \leq i \leq N, i \text { odd }}}{\bigcup}\left\{p_{4 i-3}, p_{4 i-2}, g_{4 i-1}^{1}, g_{4 i}^{1}\right\} \cup \underset{\substack{1 \leq i \leq N, i \text { even }}}{\bigcup}\left\{g_{4 i-3}^{2}, g_{4 i-2}^{2}, p_{4 i-1}, p_{4 i}\right\} \cup\left\{p_{4 N+1}\right\} .
\end{aligned}
$$

with lengths $N(3 K+Q)+\sum_{i=1}^{N} x_{2 i-1}=N(3 K+Q)+\sum_{i \in A} x_{i}=\beta$ and $N(3 K+Q)+\sum_{i \in B} x_{i}=\beta$ respectively. Consequently, both candidate longest paths are in fact longest paths, and the answer to the GCRP instance $(D, \beta)$ is like the answer to the EOP instance, viz. affirmative.

(ii). Suppose next that the answer to the GCRP instance $(D, \beta)$ is affirmative, and let $S$ be a selection of $D$ with $L(D(S)) \leq \beta$. Since $M$ is very large, $D(S)$ cannot contain any arc with length $M$. This means that, for each quadruple of layers $4 i-3,4 i-2,4 i-1$ and $4 i(i=1,2, \ldots, N)$, either all four grip nodes $g_{4 i-3}^{1}, g_{4 i-2}^{1}, g_{4 i-1}^{1}$ and $g_{4 i}^{1}$, or all four grip nodes $g_{4 i-3}^{2}, g_{4 i-2}^{2}, g_{4 i-1}^{2}$ and $g_{4 i}^{2}$ are in $S$. Therefore, $S$ can be denoted by

$$
S=\left\{g_{0}\right\} \cup \bigcup_{i=1}^{N}\left\{g_{4 i-3}^{\alpha_{i}}, g_{4 i-2}^{\alpha_{i}}, g_{4 i-1}^{\alpha_{i}}, g_{4 i}^{\alpha_{i}}\right\} \cup\left\{p_{4 N+1}\right\}
$$

with $\alpha_{i} \in\{1,2\}(i=1, \ldots, N)$. Now consider the two paths

$$
\begin{aligned}
& P_{1}^{\prime}=\left(g_{0}, g_{1}^{\alpha_{1}}, g_{2}^{\alpha_{1}}, p_{3}, p_{4}, p_{5}, p_{6}, g_{7}^{\alpha_{2}}, g_{8}^{\alpha_{2}}, g_{9}^{\alpha_{3}}, g_{10}^{\alpha_{3}}, p_{11}, p_{12}, p_{13}, p_{14}, g_{15}^{\alpha_{4}}, g_{16}^{\alpha_{4}}, g_{17}^{\alpha_{5}}, \ldots, p_{4 N+1}\right) \\
& P_{2}^{\prime}=\left(g_{0}, p_{1}, p_{2}, g_{3}^{\alpha_{1}}, g_{4}^{\alpha_{1}}, g_{5}^{\alpha_{2}}, g_{6}^{\alpha_{2}}, p_{7}, p_{8}, p_{9}, p_{10}, g_{11}^{\alpha_{3}}, g_{12}^{\alpha_{3}}, g_{13}^{\alpha_{4}}, g_{14}^{\alpha_{4}}, p_{15}, p_{16}, p_{17}, \ldots, p_{4 N+1}\right)
\end{aligned}
$$

The lengths of these paths are $L\left(P_{1}^{\prime}\right)=N(3 K+Q)+\sum_{i \in A} x_{i}$ and $L\left(P_{2}^{\prime}\right)=N(3 K+Q)+\sum_{i \in B} x_{i}$ respectively, where $(A, B)$ is a partition of $\{1, \ldots, 2 N\}$ with $\left|A \cap I_{i}\right|=\left|B \cap I_{i}\right|=1$ for $i=$ 
$1, \ldots, N$. Since both $P_{1}^{\prime}$ and $P_{2}^{\prime}$ have lengths that are at most the longest path length in $D(S)$, and since the latter on its turn is at most $\beta$, it follows that $L\left(P_{1}^{\prime}\right) \leq \beta$ and $L\left(P_{2}^{\prime}\right) \leq \beta$. These observations, combined with the choice of $\beta=N(3 K+Q)+\frac{1}{2} \sum_{i=1}^{2 N} x_{i}$ renders $\sum_{i \in A} x_{i}=$ $\sum_{i \in B} x_{i}$. Hence $(A, B)$ is an even-odd partition of $\{1, \ldots, 2 N\}$, thus establishing the fact that like the GCRP instance $(D, \beta)$, the EOP instance allows for an affirmative answer as well.

As a final comment it may be worth noticing that, as expected, the instance of GCRP that is created in the proof of Theorem 7 , does not satisfy condition (1) (thereby leaving the $P=N P$ question unanswered). Indeed, for $i=1,2, \ldots, N$, we have

$$
d\left(g_{4 i-2}^{1}, g_{4 i-1}^{1}\right)+d\left(p_{4 i-2}, p_{4 i-1}\right)=0<2 Q=d\left(g_{4 i-2}^{1}, p_{4 i-1}\right)+d\left(p_{4 i-2}, g_{4 i-1}^{1}\right)
$$

which contradicts condition (1).

\section{Conclusions}

The main contribution of this paper is a "two-phase", polynomial time dynamic programming algorithm for the Component Retrieval Problem, a problem that arises in the automated assembly of printed circuit boards. We have broadened the scope of our analysis by modelling the problem as a longest path minimization problem in a PERT/CPM-like network with design aspects. As an alternative interpretation, the problem can also be viewed as a shortest path problem with side-constraints. Both interpretations have proven to be crucial in the development and description of the proposed solution algorithm. Finally, we have sharply delineated the complexity of the problem by proving that it becomes $N P$-hard when additional structure on the activity durations in the PERT/CPM network is absent.

\section{Acknowledgements}

The first author has been partially supported in the course of this research by AFOSR (grant F4962093-1-0041), ONR (grants N00014-92-J-1375 and N00014-92-J-4083) and NATO (grant CRG 931531). 


\section{$9 \quad$ References}

[1] Ahmadi, R.H., "A hierarchical approach to design, planning, and control problems in electronic circuit card manufacturing", in Perspectives in Operations Management, R.K. Sarin (Ed.), pp. 409-429, Kluwer Academic Publishers, Dordrecht, The Netherlands (1993).

[2] Ahmadi, J., Grotzinger, S. and Johnson, D., "Component allocation and partitioning for a dual delivery placement machine", Operations Research, Vol. 36, No. 2, pp. 176-191 (1988).

[3] Ahuja, R.K., Magnanti, T.L. and Orlin, J.B., Network Flows, Prentice-Hall, Englewood Cliffs, New Jersey (1993).

[4] Ball, M.O. and Magazine, M.J., "Sequencing of insertions in printed circuit board assembly", Operations Research, Vol. 36, No. 2, pp. 192-201 (1988).

[5] Bard, J.F., Clayton, R.W. and Feo, T.A., "Machine setup and component placement in printed circuit board assembly", The International Journal of Flexible Manufacturing Systems, Vol. 6, No. 1, pp. 5-31 (1994).

[6] Burkard, R.E., Klinz, B. and Rudolf, R., "Perspectives of Monge properties in optimization", Research Report, TU Graz, Graz, Austria (1994). (To appear in Discrete Applied Mathematics.)

[7] Crama, Y., Flippo, O.E., van de Klundert, J.J. and Spieksma, F.C.R., "The assembly of printed circuit boards: A case with multiple machines and multiple board types", Working Paper, University of Limburg, Maastricht, The Netherlands (1995).

[8] Crama, Y., Oerlemans, A.G. and Spieksma, F.C.R., Production Planning in Automated Manufacturing, Lecture Notes in Economics and Mathematical Systems 414, Springer-Verlag, Berlin, Germany (1994).

[9] Garey, M.R. and Johnson, D.S., Computers and Intractability: A Guide to the Theory of NP-Completeness, W.H. Freeman, New York, New York (1979).

[10] Horak, T. and Francis, R.L., "Utilization of machine characteristics in PC board assembly", Working paper, Rutgers University, Newark, New Jersey (1995).

[11] Tang, C.S., "A max-min allocation problem: Its solutions and applications", Operations Research, Vol. 36, No. 2, pp. 359-367 (1988).

[12] Tang, C.S. and Denardo, E.V., "Models arising from a flexible manufacturing machine, part I: Minimization of the number of tool switches," Operations Research, Vol. 36, No. 5, pp. 767-777 (1988).

[13] Van Laarhoven, P.J.M. and Zijm, W.H.M., "Production preparation and numerical control in PCB assembly", The International Journal of Flexible Manufacturing Systems, Vol. 5, No. 3, pp. 187-207 (1993). 